\title{
Qing Hua Chang Yin attenuates lipopolysaccharide-induced inflammatory response in human intestinal cells by inhibiting NF- $\kappa \mathrm{B}$ activation
}

\author{
XIAO KE ${ }^{1}$, JINGTUAN CHEN $^{2}$, XIN ZHANG $^{2}$, WENYI FANG $^{1}$, CHUNBO YANG $^{1}$, \\ JUN PENG ${ }^{2}$, YOUQIN CHEN ${ }^{3}$ and THOMAS J. SFERRA ${ }^{3}$
}

\begin{abstract}
${ }^{1}$ Department of Gastroenterology, Second Affiliated Hospital of Fujian University of Traditional Chinese Medicine, Fuzhou, Fujian 350003; ${ }^{2}$ Academy of Integrative Medicine, Fujian University of Traditional Chinese Medicine, Minhou Shangjie, Fuzhou, Fujian 350122, P.R. China; ${ }^{3}$ Rainbow Babies and Children's Hospital, Case Western Reserve University School of Medicine, Cleveland, OH 44106, USA
\end{abstract}

Received January 14, 2013; Accepted April 10, 2013

DOI: $10.3892 / \mathrm{etm} .2013 .1071$

\begin{abstract}
Ulcerative colitis (UC) is a major form of inflammatory bowel disease (IBD), which is tightly regulated by the nuclear factor $\kappa \mathrm{B}(\mathrm{NF}-\kappa \mathrm{B})$ pathway. Thus, the suppression of $\mathrm{NF}-\kappa \mathrm{B}$ signaling may provide a promising strategy for the treatment of UC. Qing Hua Chang Yin (QHCY) is a traditional Chinese formulation, which has been used for a number of years to clinically treat UC. However, little is known with regard to its anti-inflammatory properties. In the present study, lipopolysaccharide (LPS)-stimulated Caco- 2 cells were used as an in vitro inflammatory model of the human intestinal epithelium to evaluate the anti-inflammatory effects of QHCY and its underlying molecular mechanisms. We observed that QHCY inhibited the inflammatory response in intestinal epithelial cells as it significantly and concentration-dependently reduced the LPS-induced secretion of pro-inflammatory TNF- $\alpha$ and IL-8 in Caco-2 cells. Furthermore, QHCY treatment inhibited the phosphorylation of $\mathrm{I} \kappa \mathrm{B}$ and the nuclear translocation of $\mathrm{NF}-\kappa \mathrm{B}$ in Caco-2 cells in a concentration-dependent manner, indicating that QHCY suppressed the activation of the $\mathrm{NF}-\kappa \mathrm{B}$ signaling pathway. Collectively, our results suggest that the
\end{abstract}

Correspondence to: Professor Chunbo Yang, Department of Gastroenterology, Second Affiliated Hospital of Fujian University of Traditional Chinese Medicine, 13 Hu Dong Zhi Road, Fuzhou, Fujian 350003, P.R. China

E-mail: ycbgzs2006@163.com

Abbreviations: QHCY, Qing Hua Chang Yin; UC, ulcerative colitis; IBD, inflammatory bowel disease; NF- $\mathrm{B}$, nuclear factor $\kappa \mathrm{B}$; TLR4, Toll-like receptor 4; LPS, lipopolysaccharide; TCM, traditional Chinese medicine

Key words: Qing Hua Chang Yin, traditional Chinese medicine, ulcerative colitis, inflammation, $\mathrm{NF}-\kappa \mathrm{B}$ pathway inhibition of NF- $\mathrm{BB}$-mediated inflammation may constitute a potential mechanism by which QHCY treats UC.

\section{Introduction}

Inflammatory bowel disease (IBD) consists of two main forms: Crohn's disease (CD) and ulcerative colitis (UC). These conditions are characterized by chronically relapsing disorders of the gastrointestinal tract (1-3). The etiology of IBD is unclear and there are no effective long-term treatments. Current treatment strategies depend on disease severity, and the majority of them are focused on attenuating disease symptoms rather than treating the disease. Furthermore, numerous currently used treatment approaches for IBD may lead to systemic immunosuppression which limits their long-term use. Therefore, there is a need for the development of new therapeutic agents. Complementary and alternative medicines (CAM), particularly herbal therapies, including traditional Chinese medicine (TCM), have received interest as they have relatively few side-effects and have been used as alternative remedies for a variety of diseases, including IBD (4-7).

The inflammatory response is highly regulated by multiple cellular signal transduction pathways, including the nuclear factor $\kappa \mathrm{B}(\mathrm{NF}-\kappa \mathrm{B})$ pathway, which is capable of being activated by various pathogens, including lipopolysaccharide (LPS). As a main component of the outer membrane of gram-negative bacteria, LPS has been hypothesized to form an important risk factor of IBD (8-10). LPS activates the host Toll-like receptor 4 (TLR4) via direct interaction, which subsequently transduces immune-related signals to the nucleus via transcription factors, including $\mathrm{NF}-\kappa \mathrm{B}(11,12)$, leading to the positive regulation of the expression of various pro-inflammatory cytokines, including TNF- $\alpha$, IL-6 and IL-8 (13-19). Therefore, suppression of the NF- $\mathrm{NB}$ pathway may provide an effective strategy for the treatment of inflammatory diseases, including IBD.

Qing Hua Chang Yin (QHCY) is a well-known traditional Chinese formulation that consists of a combination of eleven herbs, including Herba et Gemma Agrimoniae, Coptis chinensis Franch, Radix Sanguisorbae, Radix Paeoniae 
Rubra, Elettaria cardamomum, Magnolia officinalis, Artemisia capillaris Thunb, Herba Eupaatorii Fortunei, Semen Coicis, Semen Dolichoris Album and Poria cocos. Collectively, these components confer QHCY with the properties of eliminating heat and dampness, as well as strengthening the spleen to nourish vitality (known as tonifying the Spleen Qi in Chinese). Since the accumulation of toxic dampness and heat is one of the major causative factors in the pathogenesis of UC in the TCM system, heat clearing and eliminating dampness provides the basic principle behind the treatment of inflammatory diseases with QHCY. QHCY has long been used in China to clinically treat UC (20-25). However, the precise mechanism of its anti-inflammatory activity remains largely unclear. Using LPS-stimulated Caco-2 cells as an in vitro inflammatory model of the human intestinal epithelium, we evaluated the anti-inflammatory effects of QHCY and investigated its underlying molecular mechanisms.

\section{Materials and methods}

Materials and reagents. Dulbecco's modified Eagle's medium (DMEM), fetal bovine serum (FBS), penicillin-streptomycin and trypsin-EDTA were purchased from Invitrogen Life Technologies (Carlsbad, CA, USA). LPS from Escherichia coli serotype 055:B5 was purchased from Sigma-Aldrich (St. Louis, MO, USA). Antibodies for Western blot analysis were obtained from Cell Signaling Technology, Inc. (Beverly, MA, USA). All other reagents, unless otherwise stated, were obtained from Sigma Chemicals (St. Louis, MO, USA).

Preparation of QHCY. In total, $220 \mathrm{~g}$ dehydrated Herba et Gemma Agrimoniae, 33 g Coptis chinensis Franch, 100 g Radix Sanguisorbae, 110 g Radix Paeoniae Rubra, 56 g Elettaria cardamomum, $110 \mathrm{~g}$ Magnolia officinalis, $110 \mathrm{~g}$ Artemisia capillaris Thunb, $110 \mathrm{~g}$ Herba Eupaatorii Fortunei, $220 \mathrm{~g}$ Semen Coicis, $110 \mathrm{~g}$ Semen Dolichoris Album and $220 \mathrm{~g}$ Poria cocos were extracted with boiling water 3 times. The extracts were then combined and concentrated by boiling to a final volume of $1,000 \mathrm{ml}$. The final concentration of QHCY crude drug was $\sim 1.4 \mathrm{mg} / \mathrm{ml}$.

Cell culture. Human colon cancer Caco-2 cells were purchased from the American Type Culture Collection (Rockville, MA, USA). Cells (passages 20-40) were grown in DMEM containing $10 \%$ (v/v) FBS, 1,000 mg/l of glucose, $50 \mathrm{U} / \mathrm{ml}$ penicillin and $50 \mu \mathrm{g} / \mathrm{ml}$ streptomycin in a $37^{\circ} \mathrm{C}$ humidified incubator with $5 \% \mathrm{CO}_{2}$. Cells were subcultured at $85-90 \%$ confluence. Caco-2 cells usually reached confluence 3 days after seeding and differentiated into enterocyte-like cells 18-20 days post-confluence. Fully differentiated cells were used for the experiments. On the day of the experiment, the medium was removed and cells were washed twice with DMEM supplemented with $0.5 \%$ FBS.

Enzyme-linked immunosorbent assay (ELISA). Differentiated Caco-2 cells (20 days post-confluence) in 24-well plates were pre-incubated with various concentrations of QHCY for $1 \mathrm{~h}$ prior to being stimulated with $1 \mu \mathrm{g} / \mathrm{ml}$ of LPS for $24 \mathrm{~h}$. At the end of the experiment, the supernatants were collected following centrifugation of the cell culture media at
$3,000 \mathrm{x} \mathrm{g}$ for $10 \mathrm{~min}$. The secretion levels of cytokines were measured using a human TNF- $\alpha$ ELISA kit (Invitrogen Life Technologies, Camarillo, CA, USA) and a human IL-8 ELISA kit (BD Pharmingen, San Diego, CA, USA), according to the manufacturer's instructions. All samples were assayed in triplicate. Absorbance was read at $450 \mathrm{~nm}$.

Evaluation of cell viability by 3-(4,5-dimethylthiazol-2-yl)2,5-diphenyltetrazolium bromide (MTT) assay. Differentiated Caco-2 cells (20 days post-confluence) in 96-well plates were treated with various concentrations of QHCY for $24 \mathrm{~h}$. The cytotoxic effect of QHCY on the Caco-2 cells was examined using the MTT colorimetric assay. MTT $(100 \mu 1,0.5 \mathrm{mg} / \mathrm{ml}$ in PBS) was briefly added to each well and the samples were incubated for an additional $4 \mathrm{~h}$ at $37^{\circ} \mathrm{C}$. The purple-blue MTT formazan precipitate was dissolved in $100 \mu \mathrm{l}$ DMSO. The absorbance was measured at $570 \mathrm{~nm}$ using a spectrophotometer reader (Model ELX800; BioTek Instruments, Inc., Winooski, VT, USA).

Western blot analysis. Differentiated Caco-2 cells (20 days post-confluence) in 6-well plates were pre-incubated with various concentrations of QHCY for $1 \mathrm{~h}$ prior to being stimulated with $1 \mu \mathrm{g} / \mathrm{ml}$ of LPS for $30 \mathrm{mins}$. At the end of experiment, the cells were washed with ice-cold phosphatebuffered saline (PBS). The cells were then lysed with cell lysis buffer (50 mM Tris- $\mathrm{HCl}, \mathrm{pH} 7.4,150 \mathrm{mM} \mathrm{NaCl}$, $0.5 \%$ NP-40, $5 \mathrm{mM}$ EDTA, $50 \mathrm{mM} \mathrm{NaF}$ and $1 \mathrm{mM}$ PMSF) containing protease and phosphatase inhibitor (PI) cocktails. The cell lysate was centrifuged at $10,000 \mathrm{x}$ g at $4^{\circ} \mathrm{C}$ for $10 \mathrm{~min}$ and the supernatant was collected to examine the protein expression levels of IkB- $\alpha$ and p-IкB- $\alpha$. To determine NF-кB nuclear translocation, a nuclear extract was prepared: prior to lysis, the cells were incubated in $0.5 \mathrm{ml}$ hypotonic buffer A (10 mM HEPES, pH 7.9, $0.75 \mathrm{mM}$ spermidine, $0.15 \mathrm{mM}$ spermine, $0.1 \mathrm{mM}$ EDTA and $0.1 \mathrm{mM}$ EGTA) and allowed to swell at $4^{\circ} \mathrm{C}$. The cells were then sedimented at $300 \mathrm{x} \mathrm{g}$ for $10 \mathrm{~min}$. The supernatant was removed and replaced with $1 \mathrm{ml}$ fresh hypotonic buffer A plus PI cocktail. Cells were homogenized by 10-15 strokes in a Dounce homogenizer and then incubated in $5 \mathrm{ml}$ sucrose restoration buffer which was composed of $0.5 \mathrm{ml}$ hypotonic buffer B (500 mM HEPES, $\mathrm{pH}$ 7.9, $7.5 \mathrm{mM}$ spermidine, $1.5 \mathrm{mM}$ spermine, $2 \mathrm{mM}$ EDTA, $2 \mathrm{mM}$ EGTA and $10 \mathrm{mM}$ DTT) and $4.5 \mathrm{ml}$ of $7.5 \%$ sucrose). Nuclei were sedimented by centrifugation at $14,000 \times \mathrm{g}$ for $1 \mathrm{~min}$. The supernatant was removed and the pellet was resuspended in lysis buffer. Protein concentrations in total lysates or nuclear extracts were quantified using BCA. Equivalent amounts of protein were resolved in $12 \%$ sodium dodecyl sulfate-polyacrylamide gel electrophoresis (SDS-PAGE) gels and electroblotted. The PVDF membranes were blocked with $5 \%$ skimmed milk and probed with primary antibodies against IkB- $\alpha$, -IkB- $\alpha$, p50, RelA and $\beta$-actin $(1: 1,000)$ overnight at $4^{\circ} \mathrm{C}$ and then with appropriate HRP-conjugated secondary antibody followed by enhanced chemiluminescence detection.

Statistical analysis. Data were analyzed using the SPSS package for Windows (Version 11.5; SPSS, Inc., Chicago, IL, USA). Statistical analysis of the data was performed with the 

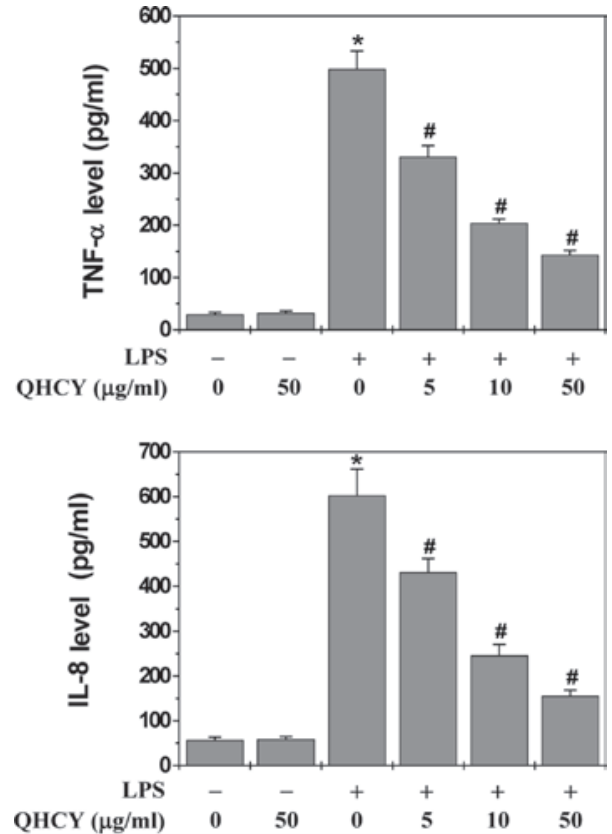

Figure 1. Effect of QHCY on the protein secretion levels of TNF- $\alpha$ and IL-8 in LPS-stimulated Caco-2 cells. Differentiated Caco-2 cells (20 days post-confluence) in 24-well plates were pre-incubated with the indicated concentrations of QHCY for $1 \mathrm{~h}$ prior to being stimulated with LPS for $24 \mathrm{~h}$. The secretion levels of TNF- $\alpha$ and IL- 8 were examined by ELISA. Data are presented as averages with SD (error bars) from at least three independent experiments. ${ }^{*} \mathrm{P}<0.05$ vs. control cells; ${ }^{*} \mathrm{P}<0.05$ vs. cells treated with LPS but without QHCY. QHCY, Qing Hua Chang Yin; LPS, lipopolysaccharide; ELISA, enzyme-linked immunosorbent assay.

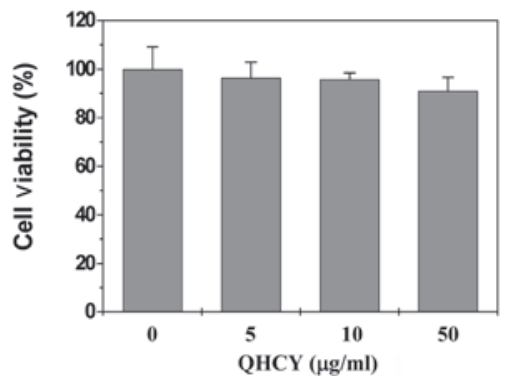

Figure 2. Effect of QHCY on Caco-2 cell viability. Differentiated Caco-2 cells ( 20 days post-confluence) in 96 -well plates were treated with indicated concentrations of QHCY for $24 \mathrm{~h}$. Cell viability was determined using the MTT assay. The data were normalized to the viability of control cells. Data are presented as averages with SD (error bars) from at least three independent experiments. QHCY, Qing Hua Chang Yin; MTT, 3-(4,5-dimethylthiazol2-yl)-2,5-diphenyltetrazolium bromide.

Student's t-test and one-way ANOVA. $\mathrm{P}<0.05$ was considered to indicate a statistically significant result.

\section{Results}

QHCY inhibits LPS-induced inflammatory response in intestinal epithelial cells. The effect of QHCY on LPS-induced inflammation in differentiated Caco- 2 cells was evaluated by measuring the secretion levels of pro-inflammatory cytokines (TNF- $\alpha$ and IL-8) since the release of cytokines is considered to represent an indicator of inflammatory response. Following pretreatment with various concentrations of $\mathrm{QHCY}$ for $1 \mathrm{~h}$,

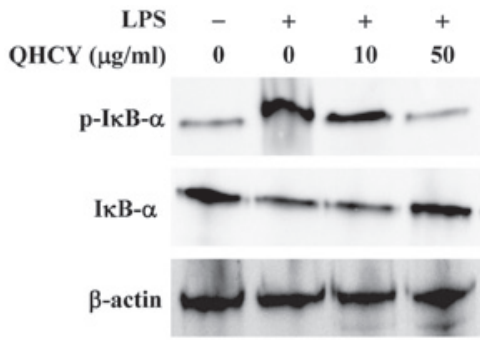

Figure 3. Effect of QHCY on LPS-mediated IкB phosphorylation in Caco-2 cells. Differentiated Caco-2 cells (20 days post-confluence) in 6-well plates were pre-incubated with the indicated concentrations of QHCY for $1 \mathrm{~h}$ prior to being stimulated with LPS for $30 \mathrm{~min}$. IкB phosphorylation was determined by Western blotting. $\beta$-actin was used as the internal control. Images are representatives of three independent experiments. QHCY, Qing Hua Chang Yin; LPS, lipopolysaccharide.

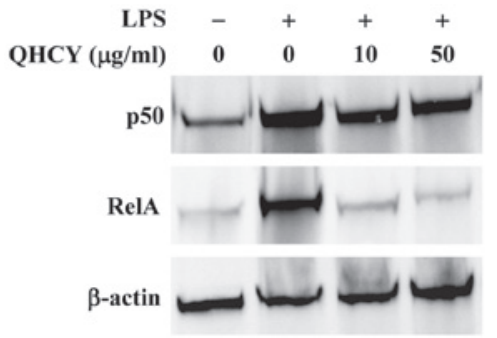

Figure 4. Effect of QHCY on LPS-mediated NF- $\kappa \mathrm{B}$ nuclear translocation in Caco-2 cells. Differentiated Caco-2 cells (20 days post-confluence) in 6-well plates were pre-incubated with indicated concentrations of QHCY for $1 \mathrm{~h}$ prior to being stimulated with LPS for 30 mins. Nuclear extracts were prepared as described in Materials and methods. The nuclear content of two subunits of NF- $\kappa \mathrm{B}$, p50 and RelA, was determined by Western blotting. $\beta$-actin was used as the internal control. Images are representatives of three independent experiments. QHCY, Qing Hua Chang Yin; LPS, lipopolysaccharide.

differentiated Caco-2 cells were stimulated with $1 \mu \mathrm{g} / \mathrm{ml}$ LPS for $24 \mathrm{~h}$ and the levels of TNF- $\alpha$ and IL-8 in the culture supernatant were assessed by ELISA. As shown in Fig. 1, LPS stimulation significantly induced the release of TNF- $\alpha$ and IL-8 in Caco-2 cells. However, QHCY treatment significantly and concentration-dependently reduced the LPS-induced secretion of TNF- $\alpha$ and IL-8, indicating that QHCY may inhibit inflammation in intestinal epithelial cells.

Cytotoxicity of $Q H C Y$ in Caco-2 cells. To exclude the possibility that the anti-inflammatory activity of QHCY was due to cytoxicity, we determined its effect on Caco-2 cell viability using the MTT assay. As shown in Fig. 2, the cell viability was not affected by treatment with QHCY and/or LPS, suggesting that the inhibitory effect of QHCY on LPS-induced inflammation in intestinal epithelial cells did not result from a cytotoxic action.

QHCY suppresses LPS-induced NF- $\mathrm{B}$ B activation in intestinal epithelial cells. NF- $\mathrm{B}$ is a critical transcription factor for inflammation response. Activation of the $\mathrm{NF}-\kappa \mathrm{B}$ pathway consists of several key processes, including the phosphorylation and degradation of $\mathrm{I} \kappa \mathrm{B}$ and the subsequent nuclear translocation of NF- $\kappa \mathrm{B}$. To investigate the mechanism of QHCY's antiinflammatory activity, we examined its effect on LPS-induced activation of the $\mathrm{NF}-\kappa \mathrm{B}$ pathway in intestinal epithelial cells. Differentiated Caco-2 cells were pretreated with QHCY for $1 \mathrm{~h}$ 
followed by stimulation with LPS for another $30 \mathrm{~min}$, and IкB phosphorylation was examined by Western blotting. As shown in Fig. 3, upon LPS stimulation, the phosphorylation level of IкB markedly increased and this increase was significantly attenuated by $\mathrm{QHCY}$ in a concentration-dependent manner.

To verify these results, we examined the change in nuclear content of two subunits of NF-kB, p50 and RelA, to evaluate the effect of QHCY on LPS-induced NF- $\mathrm{BB}$ nuclear translocation, which is an important step for NF- $\mathrm{KB}$ activation. In agreement with the observed inhibitory effect on ІкB- $\alpha$ degradation, QHCY concentration-dependently prevented the LPS-induced nuclear translocation of p50 and RelA (Fig. 4).

\section{Discussion}

As a major form of IBD, UC is the result of a chronic intestinal inflammatory response (1-3). Since the precise etiology of UC remains unknown, there are no effective long-term treatments. In addition, many currently used UC treatments lead to the development of systemic immunosuppression. Therefore, there is an urgent need for the development of new therapeutic agents. Natural products, including TCM, have received interest as they have relatively few side-effects and have been used as alternative remedies for a variety of diseases, including IBD (4-7). QHCY is a TCM formulation that has been demonstrated to be effective in China for the clinical treatment of UC (20-25). However, the molecular mechanism of its anti-inflammatory activity remains to be elucidated. Therefore, prior to the development of QHCY as an anti-UC agent, the mode of its anti-inflammatory action requires elucidation.

Pro-inflammatory cytokines produced in the intestine, including IL- 8 and TNF- $\alpha$, are important in the pathogenesis of IBD; the release of pro-inflammatory cytokines is therefore considered to represent an indicator of the inflammatory response. Using LPS-stimulated Caco-2 cells as an in vitro inflammatory model of the human intestinal epithelium, we observed that QHCY significantly and concentration-dependently reduced the LPS-induced secretion of TNF- $\alpha$ and IL-8, demonstrating that QHCY inhibited the inflammatory response in intestinal epithelial cells. The inflammatory response is tightly regulated by TLRs, a family of pattern-recognition receptors (PRRs), which enable immune systems to recognize pathogen-associated molecular patterns (PAMPs). Different TLRs recognize different PAMPs, including LPS that functions as a specific ligand for TLR4 (8-10,26-29). Following activation by ligand binding, TLR4 transduces the immune-related signals to the nucleus via transcription factors, including nuclear factor $\mathrm{\kappa B}$. As one of the most significant nuclear transcription factors, $\mathrm{NF}-\kappa \mathrm{B}$ is involved in the control of several important physiological processes, particularly the immune and inflammatory responses. In unstimulated cells, NF- $\mathrm{kB}$ is sequestered in the cytosol via interaction with inhibitory I $\kappa \mathrm{B}$ proteins. However, when cells receive pathological stimuli, IкB proteins are phosphorylated by IкB kinase (IKK). Phosphorylation of I $\kappa$ B proteins results in their ubiquitination and degradation, which subsequently releases sequestered NF- $\kappa B$, leading to its translocation to the nucleus where it induces the expression of various pro-inflammatory cytokines (15-19). Using Western blotting, we observed that QHCY treatment inhibited the phosphorylation of IкB and the nuclear translocation of $\mathrm{NF}-\kappa \mathrm{B}$ in Caco-2 cells in a concentration-dependent manner, suggesting that $\mathrm{QHCY}$ suppresses the activation of the NF- $\mathrm{\kappa B}$ signaling pathway.

In conclusion, in the present study we demonstrated that QHCY ameliorates the inflammatory response by inhibiting the activation of the NF- $\mathrm{BB}$ pathway. Our results further suggest that QHCY may be an effective traditional Chinese formulation for the treatment of UC and other inflammatory conditions.

\section{Acknowledgements}

This study was supported by the National Natural Science Foundation of China (no. 81173432).

\section{References}

1. Rezaie A, Parker RD and Abdollahi M: Oxidative stress and pathogenesis of inflammatory bowel disease: an epiphenomenon or the cause? Dig Dis Sci 52: 2015-2021, 2007.

2. Podolsky DK: Inflammatory bowel disease. N Engl J Med 347: 417-429, 2002.

3. Odashima M, Otaka M, Jin M, et al: Successful treatment of refractory duodenal Crohn's disease with infliximab. Dig Dis Sci 52: 31-32, 2007.

4. Treasure J: Herbal medicine and cancer: an introductory overview. Semin Oncol Nurs 21: 177-183, 2005.

5. Stickel F and Schuppan D: Herbal medicine in the treatment of liver diseases. Dig Liver Dis 39: 293-304, 2007.

6. Langmead L and Rampton DS: Review article: complementary and alternative therapies for inflammatory bowel disease. Aliment Pharmacol Ther 23: 341-349, 2006.

7. Bensoussan M, Jovenin N, Garcia B, et al: Complementary and alternative medicine use by patients with inflammatory bowel disease: results from a postal survey. Gastroenterol Clin Biol 30: 14-23, 2006.

8. Caradonna L, Amati L, Magrone T, Pellegrino M, Jirillo E and Caccavo D: Enteric bacteria, lipopolysaccharides and related cytokines in inflammatory bowel disease: biological and clinical significance. J Endotoxin Res 6: 205-214, 2000.

9. Böcker U, Yezerskyy O, Feick P, et al: Responsiveness of intestinal epithelial cell lines to lipopolysaccharide is correlated with Toll-like receptor 4 but not Toll-like receptor 2 or CD14 expression. Int J Colorectal Dis 18: 25-32, 2003.

10. Bruewer M, Utech M, Ivanov AI, Hopkins AM, Parkos CA and Nusrat A: Interferon-gamma induces internalization of epithelial tight junction proteins via a macropinocytosis-like process. FASEB J 19: 923-933, 2005.

11. Schreiber S, Nikolaus S and Hampe J: Activation of nuclear factor kappa B inflammatory bowel disease. Gut 42: 477-484, 1998.

12. Andresen L, Jørgensen VL, Perner A, Hansen A, Eugen-Olsen J and Rask-Madsen J: Activation of nuclear factor kappaB in colonic mucosa from patients with collagenous and ulcerative colitis. Gut 54: 503-509, 2005.

13. Lakatos PL, Fischer S, Lakatos L, et al: Current concept on the pathogenesis of inflammatory bowel disease-crosstalk between genetic and microbial factors: pathogenic bacteria and altered bacterial sensing or changes in mucosal integrity take 'toll'? World J Gastroenterol 12: 1829-1841, 2006.

14. Lu YC, Yeh WC and Ohashi PS: LPS/TLR4 signal transduction pathway. Cytokine 42: 145-151, 2008.

15. Snape WJ Jr and Kao HW: Role of inflammatory mediators in colonic smooth muscle function in ulcerative colitis. Dig Dis Sci 33 (Suppl 3): 65S-70S, 1988.

16. Neuman MG: Immune dysfunction in inflammatory bowel disease. Transl Res 149: 173-186, 2007.

17. Eckmann L, Jung HC, Schürer-Maly C, Panja A, MorzyckaWroblewska E and Kagnoff MF: Differential cytokine expression by human intestinal epithelial cell lines: regulated expression of interleukin 8. Gastroenterol 105: 1689-1697, 1993.

18. Wang D, DuBois RN and Richmond A: The role of chemokines in intestinal inflammation and cancer. Curr Opin Pharmacol 9: 688-696, 2009. 
19. Brynskov J, Foegh P, Pedersen G, et al: Tumour necrosis factor alpha converting enzyme (TACE) activity in the colonic mucosa of patients with inflammatory bowel disease. Gut 51: 37-43, 2002.

20. Wang XY and Tian DL: Etiological and pathological characteristics of ulcerative colitis and TCM differentiation and treatment. Beijing Zhong Yi Yao Da Xue Xue Bao 30: 554-559, 2007 (In Chinese)

21. Gong YP, Liu W, Ma GT, et al: Randomized control study of 'Qingchang Suppository' on ulcerative colitis. Shanghai Zhong Yi Yao Da Xue Xue Bao 21: 33-36, 2007 (In Chinese).

22. Fu NL and Huang JY: Progress of clinical research of traditional Chinese medicine for the treatment of ulcerative colitis. Journal of Traditional Chinese Medicine 40: 501-503, 1999 (In Chinese)

23. Li QG: An idea about treatment of ulcerative colitis by TCM methods. Beijing Zhong Yi 23: 149-150, 2004 (In Chinese).

24. Wang CH, Gao WY, Li YF, et al: Study of Fufangkushen colon-release capsule on ulcerative colitis of endo-retention of damp heat type. Xian Dai Zhong Xi Yi Jie He Za Zhi 18: 13-15, 2009 (In Chinese)
25. Chen JT, Ke X, Fu XY, et al: The clinical study of heat-clearing and damp-drying on the treatment of damp-heat ulcerative colitis. Zhongguo Zhong Xi Yi Jie He Xiao Hua Za Zhi 17: 256-257, 2009 (In Chinese)

26. Kawai T and Akira S: TLR signaling. Cell Death Differ 13: 816-825, 2006.

27. Johnson GB, Brunn GJ, Kodaira Y and Platt JL: Receptormediated monitoring of tissue well-being via detection of soluble heparan sulfate by Toll-like receptor 4. J Immunol 168: 5233-5239, 2002.

28. Lehnardt S, Schott E and Trimbuch T: A vicious cycle involving release of heat shock protein 60 from injured cells and activation of Toll-like receptor 4 mediates neurodegeneration in the CNS. J Neurosci 28: 2320-2331, 2008.

29. Smiley ST, King JA and Hancock WW: Fibrinogen stimulates macrophage chemokine secretion through Toll-like receptor 4. J Immunol 167: 2887-2894, 2001 